\title{
Comportamiento microestructural de una fundición de magnesio puro con control en la temperatura de solidificación
}

\author{
Microstructural behavior of a pure magnesium casting with solidification \\ temperature control.
}

\author{
Sergio Medina ${ }^{1}$, César Isaza ${ }^{1}$, Patricia Fernández-Morales ${ }^{2}$, Emigdio Mendoza ${ }^{2}$, J.M. Meza ${ }^{1}$ \\ ${ }^{1}$ Departamento de Materiales y Minerales, Universidad Nacional de Colombia, Sede Medellín, Medellín, \\ Colombia. \\ ${ }^{2}$ Facultad de Ingeniería Mecánica, Universidad Pontificia Bolivariana, Medellín, Colombia. \\ Correo-e: samedinae@unal.edu.co
}

\begin{abstract}
Resumen- En este trabajo se estudió el comportamiento microestructural del magnesio ( $\mathrm{Mg}$ ) puro luego de ser sometido a un proceso de fundición realizado en un horno con control de atmósfera de fabricación propia y con control de la temperatura de solidificación. Con este trabajo se buscó determinar el cambio en la composición del $\mathrm{Mg}$ debido a la atmosfera protectora utilizada mediante SEM/EDS y se estudió el cambio en la microestructura del $\mathrm{Mg}$ puro luego de realizar una solidificación en una lingotera a una temperatura de $200^{\circ} \mathrm{C}$ dentro del sistema de fundición, en el cuál se utilizó una atmósfera pobre en oxígeno con una mezcla de $\mathrm{CO}_{2}$ y $\mathrm{SF}_{6}$. De la evaluación composicional se evidenció un cambio en el material debido a los gases usados y los elementos con los que interactuó el Mg. Se pudo observar un cambio en la microestructura del $\mathrm{Mg}$ por medio de análisis de microscopía óptica y SEM, observando diferencias en la cantidad y distribución de inclusiones y partículas presentes en el $\mathrm{Mg}$. Además, se observó la generación de una estructura dendrítica tras el proceso de fundición, lo cual no se evidenció en el material base. Finalmente, con el sistema de fundición fabricado se logró obtener un lingote de $\mathrm{Mg}$ con condiciones similares al material base, además obtener una microestructura con granos más finos y una mejor distribución de partículas en la matriz.
\end{abstract}

Palabras clave - Fundición, Magnesio puro, Microestructura, Solidificación.

Abstract- The study of the microstructural behavior of pure magnesium (Mg) after a casting process conducted in a furnace of own manufacture with controlled atmosphere and solidification temperature control is presented. This study aimed to determine the change in the composition of the Mg by SEM/EDS, owing to the protective atmosphere used. Besides, the change in the microstructure of $\mathrm{Mg}$ was studied by performing solidification in a mold at temperature of $200^{\circ} \mathrm{C}$ within the casting system, in which a poor oxygen atmosphere was used with a mixture of $\mathrm{CO} 2$ and SF6. Through the compositional evaluation was evident a change in the material owing to the gases used and the elements that interacted with the Mg. It was possible to see a change in the microstructure of the $\mathrm{Mg}$ by optical microscopy analysis and SEM, observing differences in the amount and distribution of particles and inclusions present in the Mg. After casting process, the generation of a dendritic structure was observed, but not in the base material. Finally, using the manufactured casting system was possible to obtain an ingot of $\mathrm{Mg}$ with similar conditions to the base material, in addition to obtain a microstructure with finer grain and better distribution of particles in the matrix.

Key Word - Casting, Pure magnesium, Microstructure, Solidification.

\section{INTRODUCCIÓN}

Desde la década de los años 50, el magnesio ha tenido una importancia apreciable para el desarrollo militar, aeronáutico y aeroespacial debido a que es uno de los metales más livianos conocido, con una densidad de $1.74 \mathrm{~g} / \mathrm{cm}^{3}$ que es $35 \%$ menor que el aluminio y cuatro veces más ligero que los aceros convencionales [1,2]. El magnesio posee una estructura cristalina hexagonal compacta dando lugar a una limitada ductilidad, la cual puede ser mejorada con la adición de elementos como aluminio, zinc, manganeso, entre otros [3-6] , así como su resistencia mecánica y al creep pueden ser aumentadas considerablemente con adición de aluminio, zinc y algunas tierras raras [6-8].

Aunque la producción del magnesio ha aumentado hasta en un $80 \%$ en las últimas décadas [1], su manipulación se considera peligrosa debido a su punto de ignición durante los procesos de fundición lo que podría causar algún accidente. Con el fin de reducir los riesgos durante el proceso de fabricación por fundición con magnesio puro y sus aleaciones, se ha reportado la adición de elementos aleantes como el calcio, el cerio entre 
otros; para bajar el punto de ignición [9-11]. Así mismo, se debe evitar el contacto de la superficie con el oxígeno que promueve la ignición y sobre todo la producción de hidrogeno. De ahí entonces, la necesidad de una atmosfera protectora, para la cual se usa frecuentemente una mezcla de dióxido de carbono y hexafluoruro de azufre $\left(\mathrm{CO}_{2} \mathrm{y} \mathrm{SF}_{6}\right)$ [12]. Por otro lado, es bien conocido que las propiedades mecánicas del magnesio aumentan con el refinamiento de grano [13,14], cuyo refinamiento puedes ser logrado con procesos térmicos [15, 16], adición de carbono, calcio, zirconio entre otros, [17-19] y en este trabajo su busca controlar el efecto sobre el tamaño y distribución de los granos durante proceso de fundición de magnesio puro con enfriamiento y atmosfera controlada de $\mathrm{CO}_{2}$ y $\mathrm{SF}_{6}$, además de evaluar el efecto de la atmosfera sobre las características micro-estructurales del magnesio puro.

\section{PROCEDIMIENTO EXPERIMENTAL}

Se realizó un trabajo experimental para la obtención de un lingote de magnesio puro tras un proceso de refundición, cuyo material base usado fue magnesio puro y su composición es mostrada en la Tabla 1. Para la fundición se usó un sistema de fundición con control de atmósfera, que se muestra en la Figura 1, el cual consta de un horno cerrado con un crisol de grafito, un sistema basculante para el vaciado por gravedad, una lingotera con control de la temperatura y una cámara de control de atmósfera.

\begin{tabular}{|l|l|l|l|l|}
\hline $\mathbf{M g}$ & $\mathbf{A l}$ & $\mathbf{Z n}$ & $\mathbf{T i}$ & $\mathbf{C u}$ \\
\hline 99.12 & 0.812 & 0.0567 & 0.0037 & 0.0031 \\
\hline
\end{tabular}

Tabla 1. Composición química del magnesio puro.

El magnesio, luego de ser introducido en el crisol, se sometió a un calentamiento continuo a una tasa de aproximadamente $10^{\circ} \mathrm{C} / \mathrm{min}$ hasta los $690^{\circ} \mathrm{C}$. Cuando el horno alcanzó una temperatura cerca de $\operatorname{los} 350^{\circ} \mathrm{C}$ se permitió el flujo de una mezcla de gases con el fin de tener una atmósfera pobre en oxígeno y promover la formación de una capa protectora en la superficie del material que inhibiera la reacción del Mg. Los gases usados fueron dióxido de carbono y hexafluoruro de azufre en una proporción de $10 \mathrm{cfh}$ de $\mathrm{CO}_{2}$ y 1 cfh de $\mathrm{SF}_{6}$.

El magnesio fundió a una temperatura de $680^{\circ} \mathrm{C}$, y luego de llegar a la temperatura de $690^{\circ} \mathrm{C}$ se dejó un tiempo de sostenimiento de 10 minutos, y con el fin de homogenizar la colada se mantuvo una agitación mecánica. Posteriormente, el material fundido se vació en la lingotera precalentada a una temperatura de $200^{\circ} \mathrm{C}$ con un tiempo de sostenimiento de 10 minutos, con el fin de permitir una mejor transición en el enfriamiento del material. Finalmente, se extrajo el lingote para terminar el enfriamiento a temperatura ambiente. En la Figura 2a) se muestra el material en la lingotera luego del proceso de vaciado y en la Figura 2 b) se puede ver el lingote de magnesio luego del proceso de refundición.
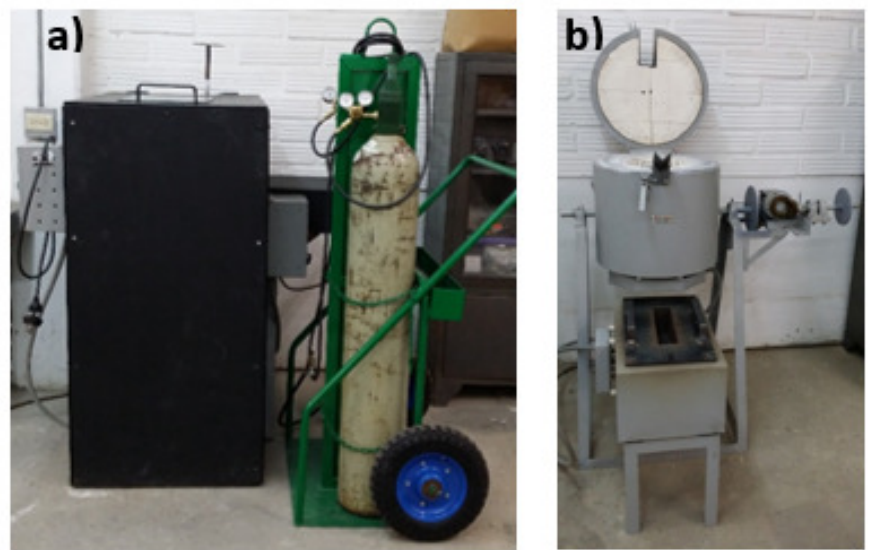

Figura 1. a) Sistema de fundición de $\mathrm{Mg}$ y a) horno basculante y lingotera.

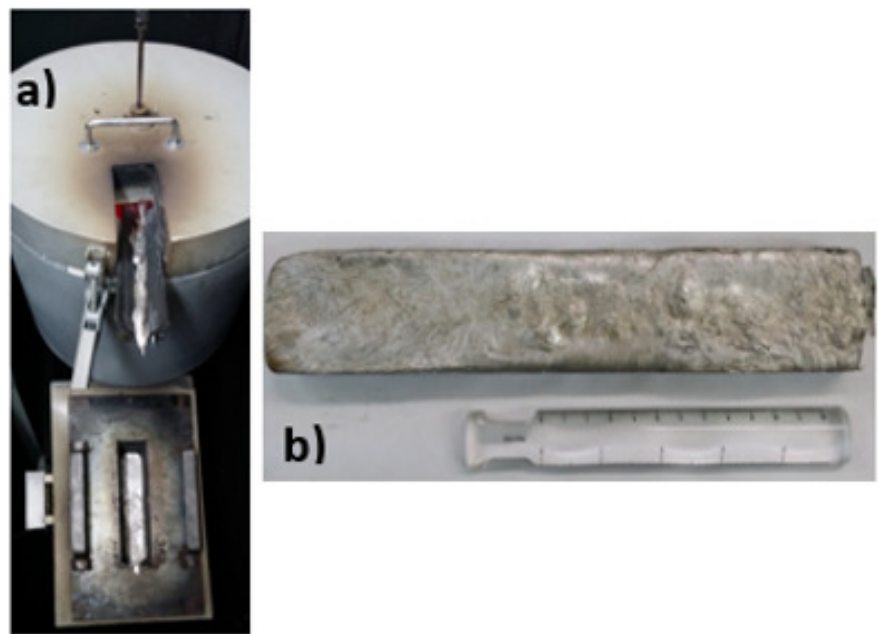

Figura 2. a) Material fundido luego de ser vaciado en la lingotera y b) Lingote de magnesio puro obtenido tras el proceso de refundición.

Tanto para el material refundido como para el material inicial, se preparó una muestra tomada de un punto al azar con el fin de realizar la caracterización y análisis del material. Ambas muestras siguieron el protocolo de preparación metalográfica según la norma ASTM-E3 para el análisis de microscopía óptica y el análisis de SEM. De forma adicional se practicaron ensayos de micro dureza bajo el procedimiento de la norma ASTM-E384.

\section{RESULTADOS Y ANÁLISIS DE RESULTADOS}

En la tabla 2 se puede observar la composición del material base refundido bajo las condiciones antes mencionadas, cuya composición no se ve afectada por el proceso de refundición del material base, lo que muestra en un principio la buena protección atmosférica durante el proceso.

\begin{tabular}{|l|l|l|l|l|}
\hline $\mathbf{M g}$ & Al & $\mathbf{Z n}$ & $\mathbf{T i}$ & $\mathbf{C u}$ \\
\hline 99.04 & 0.917 & 0.0337 & 0.0014 & 0.0032 \\
\hline
\end{tabular}

Tabla 2. Composición química del magnesio después del proceso de fundición. 
En la Fig. 3 se muestran las imágenes de la caracterización metalográfica para el magnesio base. Su microestructura revela una estructura relativamente homogénea con granos no equiaxiales, aunque bastante grandes, lo que sugiere que fue colado a altas temperaturas. De las imágenes de microscopia electrónica de barrido (SEM) mostradas en la Fig. 3c) y 3d) se puede ver el límite de grano para la microestructura del magnesio base y no sugiere la formación de algún tipo de fase hacia los límites de grano, aunque en algunas zonas se encontraron algunas partículas como se muestra en la Fig. 3d) cuya caracterización por EDS (Fig. 4) muestra un alto contenido de carbono, calcio, hierro entre otros, lo que probablemente sea generado durante la fundición y colado.
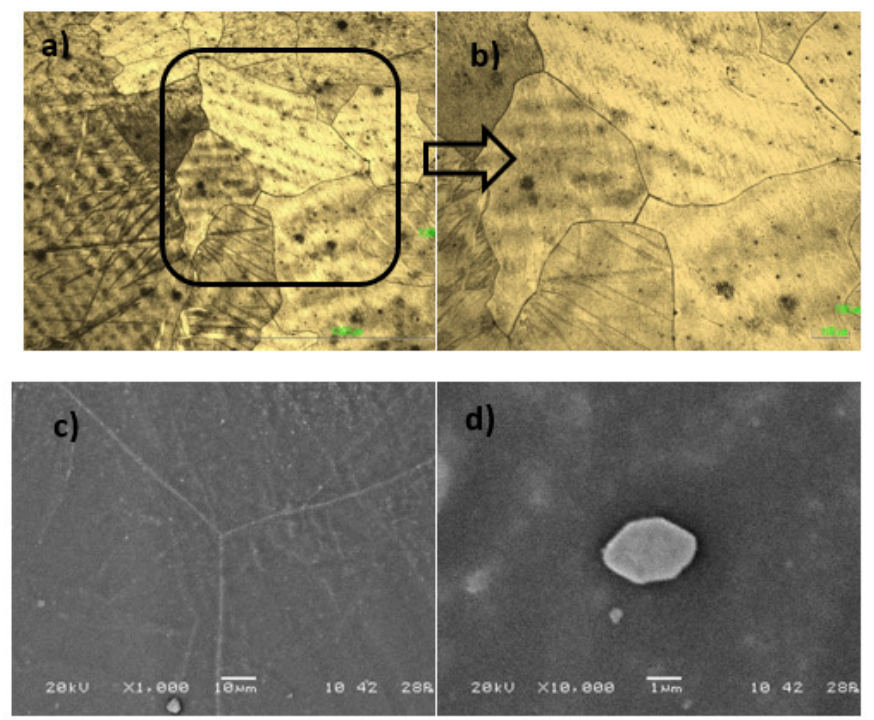

Figura 3. Microestructuras del magnesio base. a) y b) imágenes de MO del magnesio base, c) y d) imágenes de SEM del magnesio base.

\begin{tabular}{|c|c|}
\hline Element & $\begin{array}{c}\text { Weight } \\
\%\end{array}$ \\
\hline $\mathrm{C}$ & 9.39 \\
\hline $\mathrm{O}$ & 21.17 \\
\hline $\mathrm{Mg}$ & 57.88 \\
\hline $\mathrm{Si}$ & 1.20 \\
\hline $\mathrm{Cl}$ & 1.47 \\
\hline $\mathrm{Ca}$ & 4.68 \\
\hline $\mathrm{Fe}$ & 2.34 \\
\hline $\mathrm{Au}$ & 1.87 \\
\hline Totals & $\mathbf{1 0 0 . 0}$ \\
\hline
\end{tabular}

a)

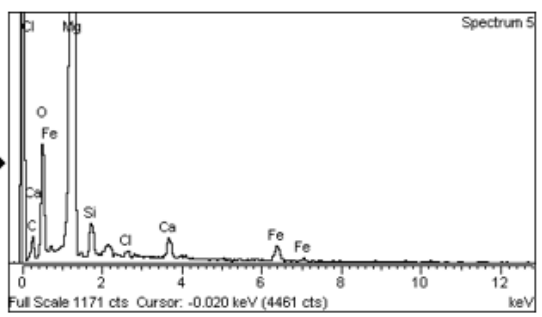

b)
Figura 4. Análisis elemental por EDS del magnesio base.

Las microestructuras del material refundido se pueden ver en las imágenes de la Figura 5. En la figura 5a) se puede ver que el magnesio tiene un límite de grano sin la evidencia de algún tipo de reacción o formación de fase debido a la presencia de los gases de protección usados para la fundición.
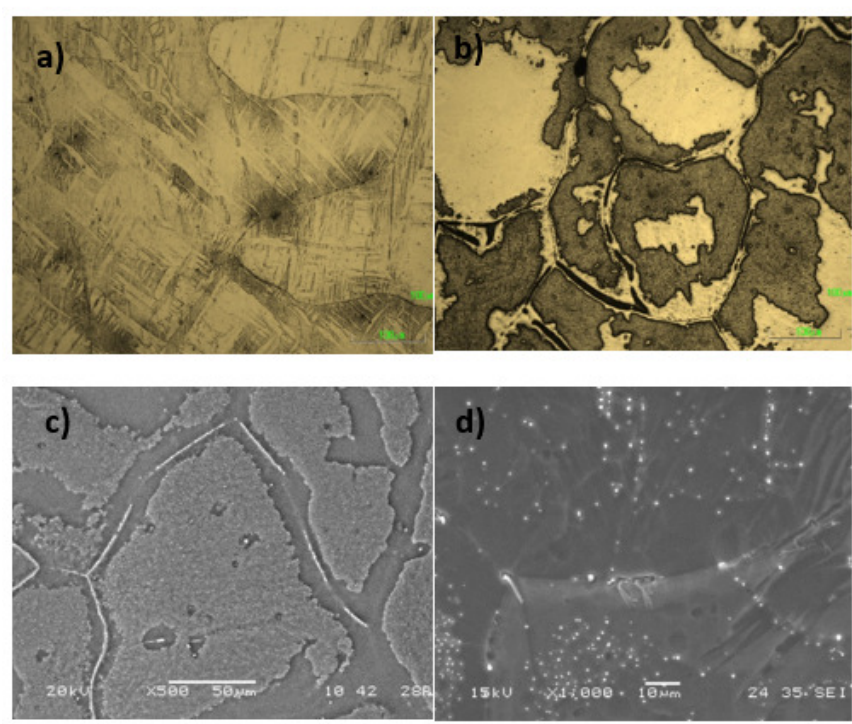

Figura 5. Microestructuras del magnesio fundido. a) y b) imágenes de MO del magnesio fundido, c) y d) imágenes de SEM del magnesio fundido.

Sin embargo, en las imágenes de la Fig. 5b) y c) se nota que hacia los límites de granos hay una coloración distinta que sugiere la formación de algún tipo de fase eutéctica, pero debido a las condiciones iniciales de material base, esto no sería posible debido a las pocas proporciones de aleantes que tiene el magnesio base; por lo que se sugiere que es una reacción química entre los límites de grano y el reactivo usado para revelar su microestructura.

De igual forma que el material base inicial, durante el análisis por SEM se evidenciaron algunas partículas cuyo análisis por EDS (Fig. 6) muestra elementos como el cloro, lo que sugiere algún tipo de impureza durante la fundición y colado del material.

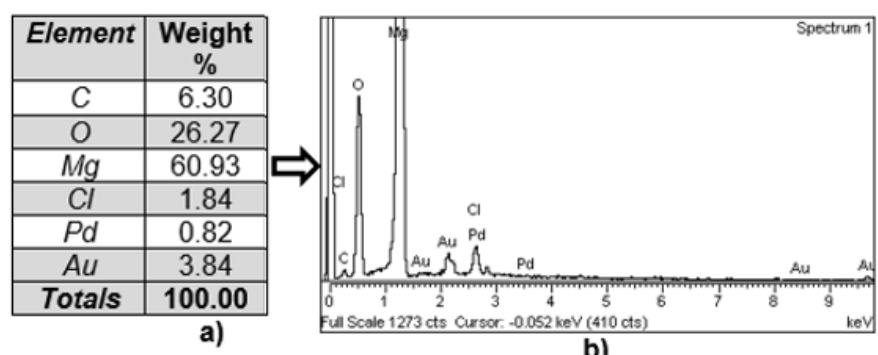

b)

Figura 6. Análisis elemental por EDS del magnesio fundido.

De los ensayos de microdureza realizados para el magnesio base y para el magnesio refundido mostrados en la Fig. 7a), se puede ver que la dureza no tiene variación, lo cual es debido, a que no hay elementos aleantes que puedan formar algún tipo de precipitado o fase en los límites de grano que cambien sus propiedades. De igual forma, se nota claramente en cuanto a dureza, que los gases usados para la fundición del magnesio no tienen un efecto sobre las propiedades mecánicas del material, lo que supone que no hubo ninguna reacción entre el magnesio y los gases usados. Sin embargo, debido a las condiciones de 
temperatura usada en la lingotera para el magnesio refundido se nota una disminución en el tamaño de grano que pasa de aproximadamente 400 a 130 micrómetros como se muestra en la gráfica de la Fig. 7b). Sin embargo, como el grano sigue siendo demasiado grande no hay un efecto sobre la dureza y por eso presentan valores parecidos para ambos materiales.

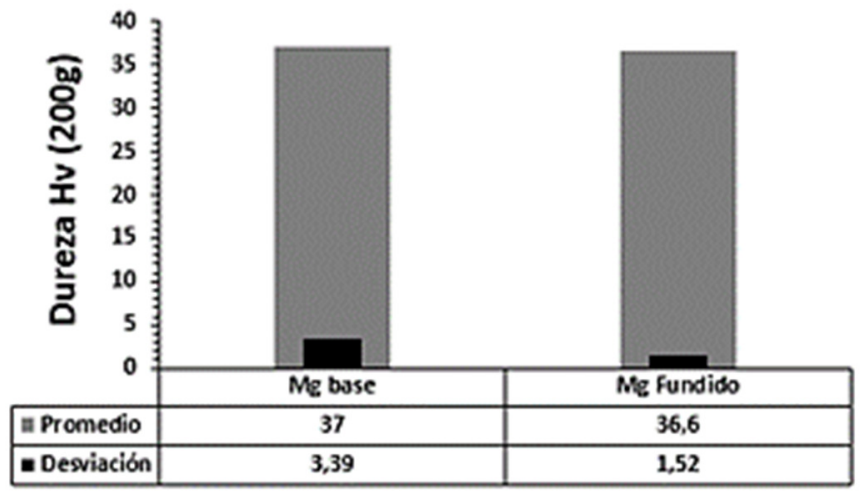

a)

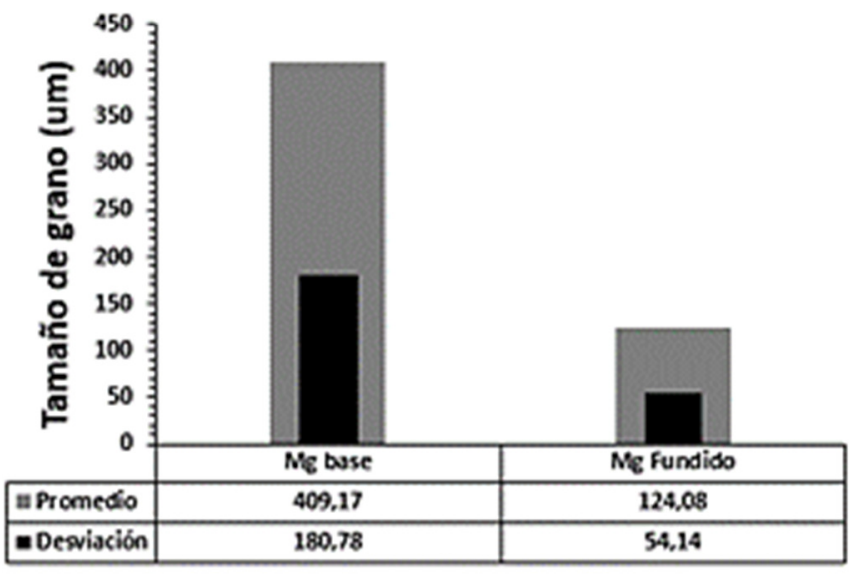

b)

Figura 7. a) Gráfica de microdurezas y b) gráfica del tamaño de grano para el magnesio base y el magnesio fundido.

\section{CONCLUSIONES}

En este trabajo se estudió la fundición de magnesio por medio de un horno eléctrico con control de atmosfera con una mezcla de los gases dióxido de carbono y hexafluoruro de azufre, con los cuales se evidenció que no hay un efecto sobre la microestructura y sobre las propiedades mecánicas del magnesio. De igual forma se logró evidenciar el buen resultado obtenido al usar la lingotera con control de temperatura para reducir el choque térmico durante el vaciado.

De igual forma, en el trabajo realizado se evidencia que la fundición del magnesio se hace viable para trabajos futuros en el desarrollo de aleaciones livianas de base de magnesio para aplicaciones estructurales en al área de la ingeniería en la industria aeronáutica y automotriz. Lo que implicaría un avance en la producción de este tipo de aleaciones en nuestra región.

\section{AGRADECIMIENTOS}

Los autores agradecen a la Universidad Pontificia Bolivariana, a la Universidad Nacional- sede Medellín, y a Colciencias quien ha financiado este estudio por medio del proyecto código 1210-569-34713.

\section{REFERENCIAS}

[1]. Kulekci, M.K., "Magnesium and its alloys applications in automotive industry". The International Journal of Advanced Manufacturing Technology, vol. 39, 9-10, p. 851-865, 2008.

[2]. Luo, A.A., "Magnesium casting technology for structural applications". Journal of Magnesium and Alloys, vol. 1, 1, p. 2-22, 2013.

[3]. Park, S.H., et al., "Magnesium alloy having high ductility and high toughness, and preparation method thereof". US Patent. Google Patents, 2014.

[4]. Sabokpa, O., A. Zarei-Hanzaki, and H. Abedi, "An investigation into the hot ductility behavior of AZ81 magnesium alloy". Materials Science and Engineering: A, vol. 550, p. 31-38, 2012.

[5]. Wang, J., et al., "A high strength and ductility $\mathrm{Mg}$ $\mathrm{Zn}-\mathrm{Al}-\mathrm{Cu}-\mathrm{Mn}$ magnesium alloy". Materials \& Design, vol. 47, p. 746-749, 2013.

[6]. Yuan, W., S.K. Panigrahi, and R.S. Mishra, “Achieving high strength and high ductility in friction stirprocessed cast magnesium alloy". Metallurgical and Materials Transactions A, vol. 44, 8, p. 3675-3684, 2013.

[7]. Xiao, W., et al., "The influence of $\mathrm{Zn}$ additions on the microstructure and creep resistance of high pressure die cast magnesium alloy AE44”. Materials Science and Engineering: A, vol. 539, p. 177-184, 2012.

[8]. Zhu, S., et al., "Evaluation of Magnesium Die-Casting Alloys for Elevated Temperature Applications: Microstructure, Tensile Properties, and Creep Resistance". Metallurgical and Materials Transactions A, p. 1-12, 2015

[9]. Czerwinski, F., "Controlling the ignition and flammability of magnesium for aerospace applications". Corrosion Science, vol. 86, p. 1-16, 2014.

[10]. Ding, J., et al. "Study of $\mathrm{Ca}$ and $\mathrm{Ce}$ Additions on Different Ignition Resistance Behavior of Magnesium Alloy". En: Materials Science Forum. Trans Tech Publ., 2014.

[11]. Liu, M., et al., "The ignition temperature of $\mathrm{Mg}$ alloys WE43, AZ31 and AZ91". Corrosion Science, vol. 54, p. 139-142, 2012.

[12]. Rongshi, C., et al., "Consideration of castability and formability for new magnesium alloys". Open Journal of Metal, 2012.

[13]. Razavi, S., et al., "Effect of grain size on prismatic slip in Mg-3Al-1Zn alloy". Scripta Materialia, vol. 67, 5, p. 439-442, 2012. 
[14]. Chen, Q., et al., "Microstructure development and tensile mechanical properties of $\mathrm{Mg}-\mathrm{Zn}-\mathrm{RE}-\mathrm{Zr}$ magnesium alloy”. Materials \& Design, vol. 40, p. 488-496, 2012.

[15]. Guo, H.-M., et al., "Refining microstructure of AZ91 magnesium alloy by introducing limited angular oscillation during initial stage of solidification". Materials Science and Engineering: A, vol. 532, p. 221-229, 2012

[16]. Liu, Y., et al., "Effects of grain size on the corrosion resistance of pure magnesium by cooling ratecontrolled solidification". Frontiers of Materials Science, vol. 9, 3, p. 247-253, 2015.

[17]. Zhang, B., et al., "Effects of calcium on texture and mechanical properties of hot-extruded $\mathrm{Mg}-\mathrm{Zn}-\mathrm{Ca}$ alloys". Materials Science and Engineering: A, vol. 539, p. 56-60, 2012.

[18]. StJohn, D., et al., "Grain refinement of magnesium alloys: a review of recent research, theoretical developments, and their application". Metallurgical and Materials Transactions A, vol. 44, 7, p. 29352949, 2013.

[19]. Robson, J. and C. Paa-Rai, "The interaction of grain refinement and ageing in magnesium-zinc-zirconium (ZK) alloys”. Acta Materialia, vol. 95, p. 10-19, 2015. 